\title{
Particle sizing in the electrodynamic balance
}

\author{
Gideon Sageev, John H. Seinfeld, and Richard C. Flagan \\ Department of Chemical Engineering, California Institute of Technology, Pasadena, California 91125
}

(Received 18 November 1985; accepted for publication 29 January 1986)

\begin{abstract}
We report a new technique for sizing particles in the electrodynamic balance. In this technique, the trajectory of a falling particle is followed with a photomultiplier tube. Particle velocities are measured by placing a mask between the particle and the detector. The masked region in the particle trajectory is roughly $0.6 \mathrm{~mm}$ wide. Output from the PMT is sampled every millisecond by an $A / D$ converter and stored in a computer. Flight times of several hundred milliseconds are measured and the size is then computed from the particle's terminal velocity. With a modification of the mask, the technique is used to verify the uniformity of the electric field through which the particle is falling. In the present work we use the technique to determine size of polystryrene latex microspheres having nominal diameters of 10 and $20 \mu$. The technique can be used on any size particle, independent of its charge-to-mass ratio, and provides the size information in a short time.
\end{abstract}

\section{INTRODUCTION}

The electrodynamic balance is an instrument in which single micron-sized particles can be levitated and studied. The historical development of the electrodynamic balance, as well as the behavior of charged particles in its electric field, has been documented by several authors. ${ }^{1-3}$ This device has found application in many research areas such as photoemission from single particles, ${ }^{4}$ energy transfer in single droplets, ${ }^{5}$ particle laser heating, ${ }^{6}$ condensation rates, ${ }^{7.8}$ and aerosol spectroscopy. ${ }^{9}$ In some cases the analysis of the experimental data obtained from the electrodynamic balance requires an estimate of the particle size. Numerous sizing techniques have been used in the past, for example the sedimentation method, the matching of scattered light from the particle to that predicted by Mie theory, the "springpoint" method, ${ }^{10}$ and the electron stepping technique. ${ }^{11}$

Among the sizing methods sedimentation is probably the oldest. The sedimentation method requires measurement of the terminal velocity of a moving particle, from which the particle radius can be calculated. This technique has the advantage that it is independent of both the electric field and the particle's charge. However, for particles on the order of $10 \mu$ and falling distances of order $1 \mathrm{~mm}$ (typical values in the present work), the uncertainty in the fall time becomes large when measured manually. This shortcoming was the impetus for the development of a new technique in which the particle trajectory is followed electronically. This technique, which is reported here, reduces the uncertainty of the fall time measurement from that carried out manually and thus provides an accurate and reproducible sizing method for particles in the electrodynamic balance.

\section{EXPERIMENTAL SYSTEM}

A detailed description of the electrodynamic balance has been provided earlier. ${ }^{8}$ During an experiment, a charged particle is levitated between a set of hyperbolic electrodes, the schematic diagram of which is shown in Fig. 1. There are two endcap electrodes between which a dc potential is held, as well as a ring electrode to which an ac voltage is applied.
The dc potential across the endcaps balances the particle against gravity, while the ac voltage on the ring provides for the focusing of the particle towards the center of the chamber. The particle is illuminated by a $\mathrm{HeNe}$ laser beam that traverses the chamber vertically through holes drilled into the center of the endcap electrodes. As shown, the $90^{\circ}$ scattered light from the particle is observed through holes in the ring electrode.

At the geometric center of the device there is no ac field. Therefore, when the electric force produced by the dc field just balances the gravitational force on the particle, the center of the chamber becomes a stable equilibrium point for the particle. Stated differently, whenever a balanced particle drifts away from the center it "feels" a net force towards the center of the chamber. For a balanced particle in the apparatus,

$$
q C\left(V_{\mathrm{dc}} / 2 Z_{0}\right)=m g
$$

where $q$ and $m$ are the particle charge and mass, $g$ is the gravitational constant, $Z_{0}$ is the distance between the center of the chamber and the endcaps (which equals $4.49 \mathrm{~mm}$ in our case), and $C$ is a geometric constant for the hyperbolic geometry of Fig. 1. The theoretical value of $C$ has been evaluated both numerically ${ }^{11}$ and analytically. ${ }^{10}$ The numerical result was found to be 0.8 , however, the calculation did not take into account the holes in the ring electrode. The analytical solution neglected the presence of the ring electrode and yielded a value for $C$ which is $\approx 10 \%$ larger than the numerical result. Since the holes in the electrodes distort the field somewhat ${ }^{10}$ from that in their absence, it is necessary to determine $C$ experimentally.

We have determined the value of cell constant ${ }^{8}$ by first measuring the size of PSL particles with the spring-point method ${ }^{10}$ using the theoretical cell constant of 0.8 . We then manually measured the fall rate of the same particles and evaluated their size, as described below, independent of the value of $C$. Knowing the size of the particles, the value of $C$ was varied until the sizes obtained by both methods were equal. The experimental value of $C$ is 0.71 , about $10 \%$ lower than the theoretical value. 


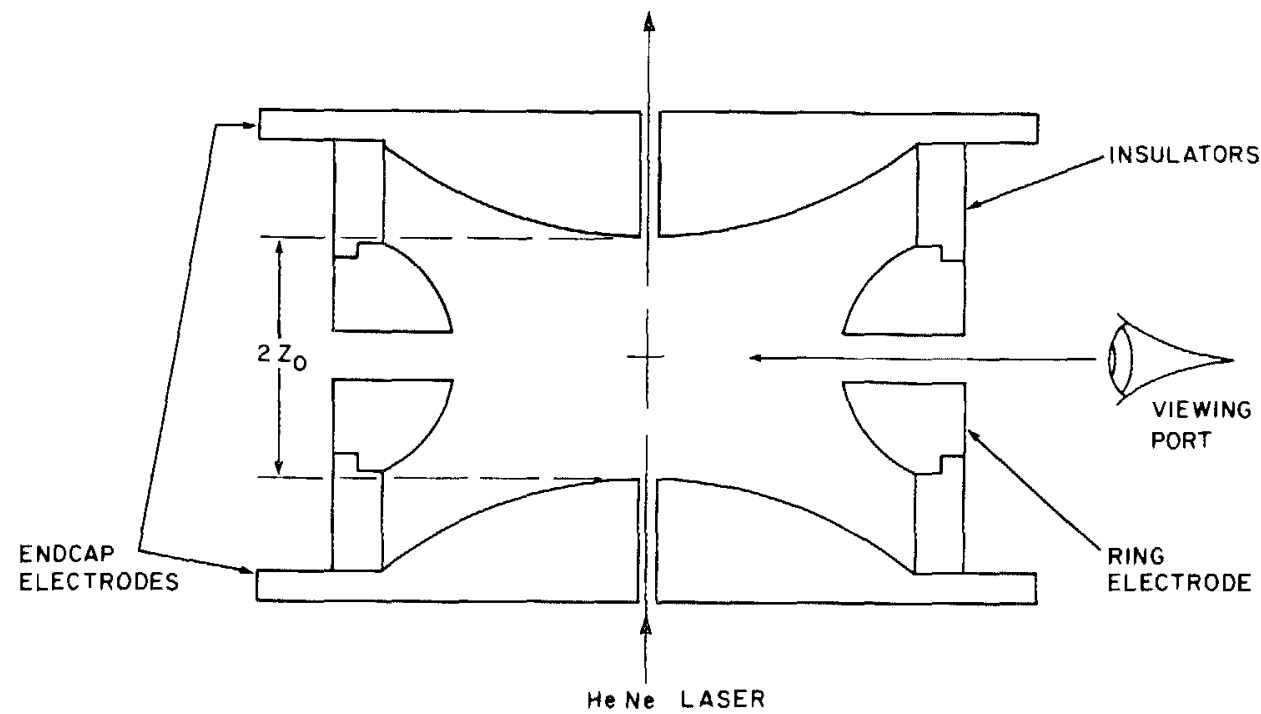

FIG. 1. Schematic diagram of the electrodynamic balance.

When using the sedimentation method, the particle's initial balancing voltage $V_{\mathrm{dc}}^{0}$ is first changed to a new value $V_{\mathrm{dc}}^{\prime}$. The ac field is then turned off and the particle begins to move under the combined influence of gravity and the electric field. In this case a force balance on the particle yields ${ }^{8}$

$$
R_{p}=\left[\frac{9 \mu v}{2 C_{c} \rho_{p} g}\left(\frac{V_{\mathrm{dc}}^{0}}{V_{\mathrm{dc}}^{0}-V_{\mathrm{dc}}^{1}}\right)\right]^{1 / 2},
$$

where $R_{p}$ and $v$ are the particle radius and terminal velocity, $C_{c}$ is the Cunningham correction factor to Stokes drag, and $\mu$ is the gas viscosity. It should be pointed out that when using this method the particle is only allowed to fall about 1 $\mathrm{mm}$ around the center of the chamber where the field is relatively uniform.

It can be shown that the time constant for a falling particle to reach terminal velocity is $m / C_{d}$, where $C_{d}$ is the drag coefficient. Using Stoke's law to compute $C_{d}$, a 20- $\mu$ polysty- rene latex sphere has a time constant of $\approx 1 \mathrm{~ms}$. In the present study all particles were allowed to fall $100-200 \mathrm{~ms}$ prior to measuring their velocity, thereby ensuring that the terminal velocity was reached.

The method by which the particle motion is detected is shown in Fig. 2. In the first step the particle is brought into the focus of a $45 \times$ microscope. The microscope eyepiece contains a reticle on which a $0.916-\mathrm{mm}$-wide strip of opaque material is placed. The strip was formed by photolithography where a precoated glass plate was first illuminated by UV light and then treated by a negative photoresist solution. The edges of the mask were observed under a microscope and found to have less than $1-\mu$ fluctuations. The particle fall distance, masked by this strip, was calibrated by focusing the microscope on a reticle scale, having $0.01-\mathrm{mm}$ divisions. Thus when a particle is focused on the mask, the distance it travels crossing the mask is known.

As shown in Fig. 2, the time it takes the particle to cross

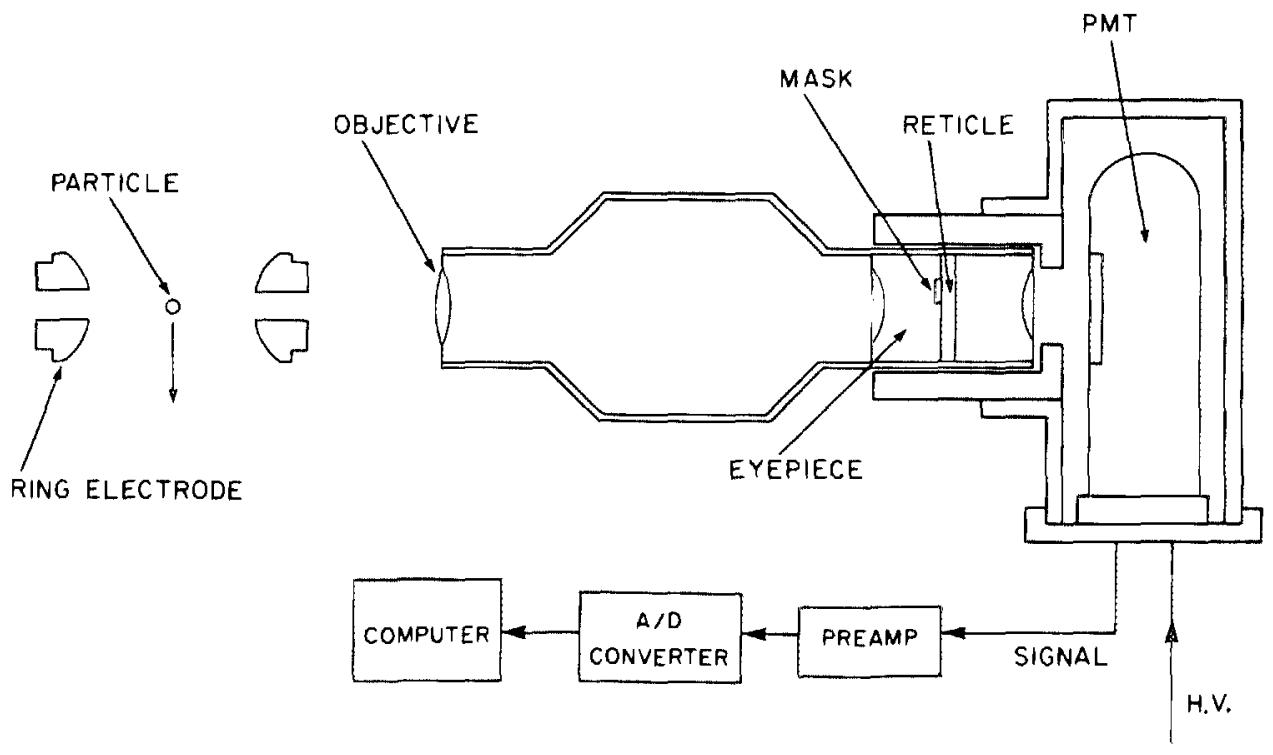

FIG. 2. Experimental arrangement for measuring particle fall times. 
the mask is measured by placing a photomultiplier tube behind the eyepiece and recording the scattered light signal from the particle. The photomultiplier tube we used is an RCA 4818 operated at about $750 \mathrm{~V}$. The scattered light signal was first preamplified and then fed into a 16-bit $A / D$ converter (Burr Brown ADC76KG). The A/D converter was triggered to convert every millisecond and its parallel output was latched and stored directly into the memory of a Zenith Z-120 computer.

The computation of the velocity from the time measurement relies on the assumption that the field is uniform near the center of the chamber. Clearly, if the field is not uniform, the particle velocity will not remain constant as the particle falls. In order to determine the uniformity of the field, a second mask was prepared, consisting of two thin strips of aluminum $(0.297 \mathrm{~mm}$ wide $)$, separated by a $0.297-\mathrm{mm}$ gap. Like the previous mask, this mask was prepared by photolithography. Since the two masked areas are equal in width, yet spatially separated from each other, a measurement of the time it takes a particle to cross each mask gives a clear indication of the field uniformity. Additionally, the signal from a particle passing through this mask yields the falling time over six different intervals. [A mask with $n$ opaque strips would have $(2 n-1)$ ! separate intervals]. By normalizing the fall time over the different intervals to that over a single strip, one obtains an accurate average of the time it takes a particle to cross each strip.

\section{月. RESULTS}

The scattered light signal obtained from a spherical (nominally $19.1 \mu$ ) polystyrene latex particle is shown in Fig. 3. The first and second steep changes in the signal correspond to the times when the particle was first obscured by the front edge of the mask and when it reappeared on the other side, respectively. The other sharp dip in the signal is caused by the particle's quick return to the center of the electrodynamic balance once the ac field is turned back on.

An expanded view of the area of interest is shown in Fig. 4. As seen in Fig. 4, the scattered light signal does not drop instantaneously as the particle moves behind the mask. This is due to the finite size of the particle. The question then arises as to which point along the steep portion of the curve

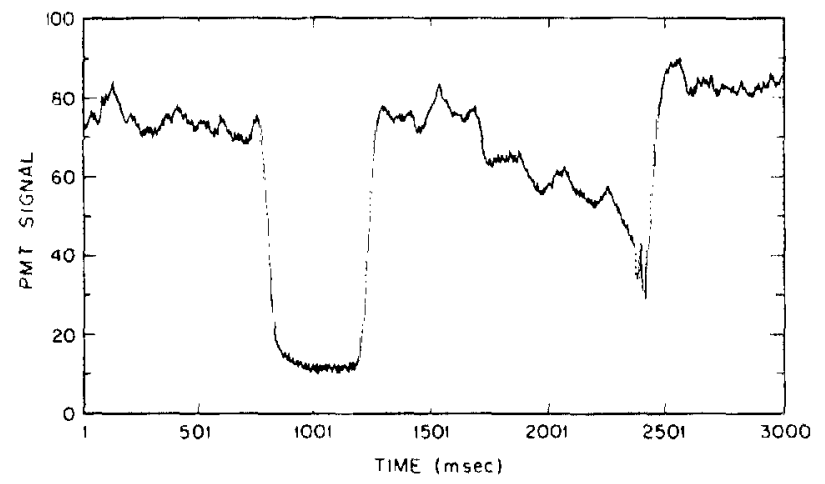

Fig. 3. Scattered light signal from a $19.5-\mu$ polystyrene latex particle crossing an opaque strip ( $0.9 \mathrm{~mm}$ wide).

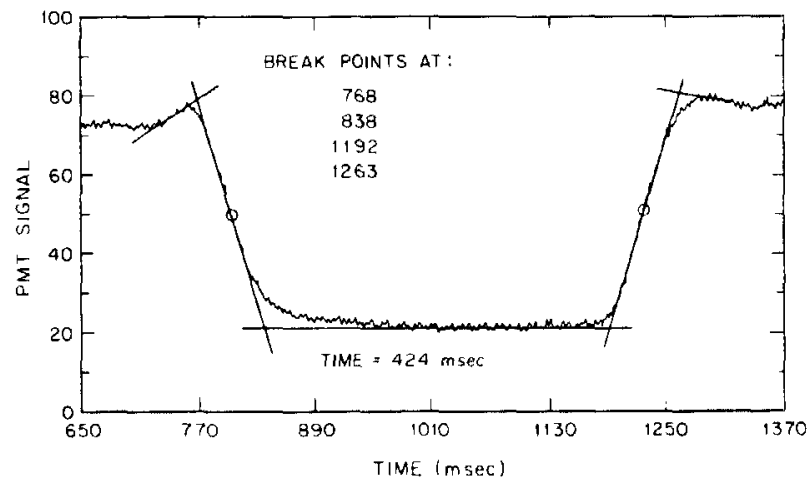

FIG. 4. Expanded view of the region where the 19.5- $\mu$ particle crosses the mask, and determination of the particle fall time by a least squares fit of the data.

should be taken as the cross-over point (by cross over we mean the time at which half the particle is behind the mask ). For the purpose of calculation we assume that the cross over occurs midway along the steep parts of the curve. The points along each of the steep parts as well as the bottom and shoulder regions were fit to straight lines by a least-squares program. The lines thus generated were plotted and the four intersection points of the five straight lines were computed. The fall time is then computed from the midpoints of the two steep lines. The terminal velocity was then found from the fall time and distance, from which the particle diameter was computed. It should be pointed out that the mask is not $100 \%$ opaque, especially near the edges; this gives rise to the rounded corners around the break points of the scattered light signal.

In order to test the assumption about the cross-over point we also measured the size of some particles by the spring-point method. When using the spring-point method, the ac trapping voltage is increased to the point at which the particle becomes unstable and begins to oscillate at half the driving frequency. ${ }^{2,10}$ We have found that the diameter resulting from the spring-point method was 2-3\% larger than that computed with the current technique. Since the velocity is proportional to the square of the particle diameter the larger size inferred from the spring-point measurement

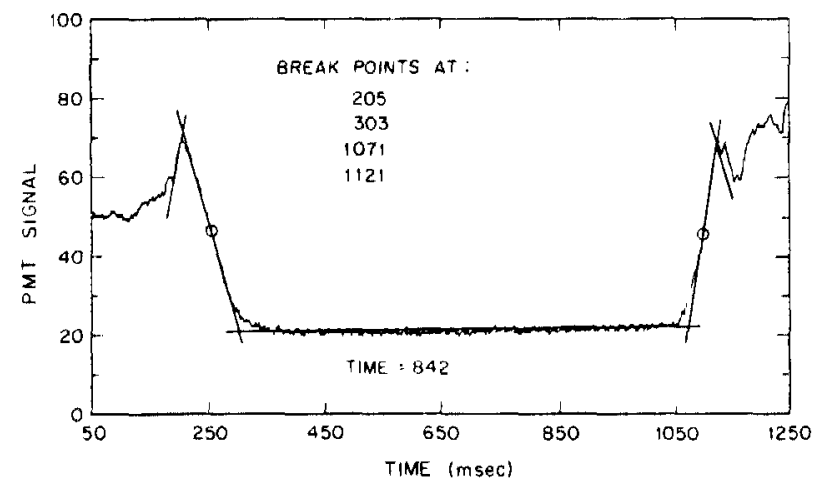

FiG. 5. Scattered light signal and fall time of a $10.15-\mu$ polystyrene latex particle. 


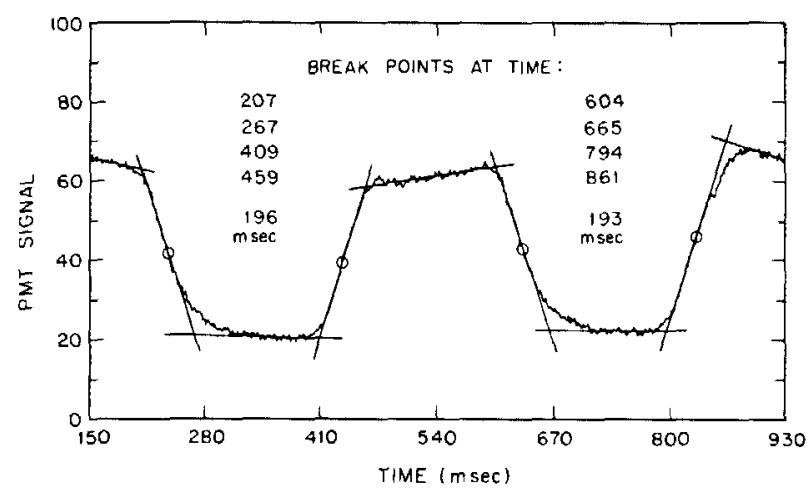

FIG. 6. Expanded signal from a $19.5 \cdot \mu$ PSL particle crossing a mask with two opaque strips (each $0.3 \mathrm{~mm}$ wide).

would correspond to a $4-6 \%$ higher velocity. The difference between the two results is partially due to the uncertainty in the earlier determination of the cell constant; however, it highlights a limitation in the precision of the current technique.

The voltage offset for the particle of Figs. 3-5 was about $12 \%$ and the resulting diameter was $18.9 \mu \mathrm{m}$. In Fig. 5 we show the scattered light signal from a $9.96-\mu \mathrm{m}$ polystyrene latex particle. (The nominal diameters of the particles were 19.1 and $9.6 \mu \mathrm{m}$ with $5.8 \%$ standard deviation.) Even though the offset voltage of $\approx 22 \%$ was used on this particle, the fall time is longer than that of the larger particle. This is a result of the drag being inversely proportional to the square of the diameter.

It must be mentioned that particle sizes can also be obtained accurately by fitting the angular scattered light from the particle to that predicted by Mie theory. ${ }^{12}$ We have not used this technique due to the geometric limitations of our present chamber. Although the Mie scattering technique is more precise than the present sizing method, the former technique only applies for homogeneous spheres whereas the method presented here could also be applied to nonspherical particles provided the shape correction factor to Stokes law is available. Additionally, the Mie scattering method re- quires knowledge of the particle's refractive index, whereas the current method is independent of this parameter.

The scattered light from a particle crossing the double mask reticle is shown in Fig. 6. Using all six time measurements in Fig. 6, we obtain an average time of $197 \mathrm{~ms}$ with a standard deviation of $1.2 \%$. Therefore, the assumption of uniform field near the center of the chamber is valid with an uncertainty of about $1 \%$. This variation is consistent with that predicted for electrodes having no holes.

Referring again to Fig. 3, it is evident that there are some small peaks in the scattered light signal on both sides of the mask. These peaks may be caused by diffraction effects and may, therefore, also contain particle size information. This additional structure does not directly affect our current results.

\section{ACKNOWLEDGMENTS}

This work was supported by U.S. Environmental Protection Agency Grant R-810857. The authors wish to thank John Lee and Wade Regehr of Caltech for their help during this project.

${ }^{1}$ E. J. Davis, Aerosol. Sci. Tech. 2, 121 (1983).

${ }^{2}$ R. H. Frickel, R. E. Shaffer, and J. B. Stamattoff, Technical Report ARCSL-TR-77041, U.S. Army Armament Research and Development Command, Chemical Systems Laboratory, Aberdeen Proving Ground, Maryland, 1978.

${ }^{3}$ R. F. Weurker, H. Shelton, and R. V. Langmuir, J. Appl. Phys. 30, 342 (1959).

${ }^{4}$ S. Amold and N. Hessel, Rev. Sci. Instrum. 56, 2066 (1985).

${ }^{5}$ L. M. Folan, S. Arnold, and S. D. Druger, Chem. Phys. Lett. 118, 322 (1985).

${ }^{6}$ R. E. Spjut, A. F. Sarofim, and J. P. Longwell, Langmuir 1, 355 (1985).

${ }^{7}$ E. J. Davis and A. K. Ray, J. Aerosol Sci. 9, 411 (1978).

${ }^{8} \mathrm{G}$. Sageev, R. C. Flagan, J. H. Seinfeld, and S. Arnold, J. Colloid Interface Sci. (in press).

${ }^{9}$ S. Arnold, E. K. Murphy, and G. Sageev, Appl. Opt. 24, 1048 (1985).

${ }^{10}$ E. J. Davis, Langmuir, 1, 379 (1985)

"M. A. Phillip, F. Gelbard, and S. Amold, J. Colloid Interface Sci. 91, 507 (1983).

${ }^{12}$ R. Chang and E. J. Davis, Langmuir, J. Colloid Interface Sci. 54, 352 (1976). 